\title{
Trajectory retrieval and component investigations of the southern polar stratosphere based on high-resolution spectroscopy of the totally eclipsed moon surface
}

\author{
Oleg S. Ugolnikov ${ }^{\mathrm{a}, *}$, Anna F. Punanova ${ }^{\mathrm{b}}$, Vadim V. Krushinsky ${ }^{\mathrm{b}}$ \\ a Space Research Institute, Russian Academy of Sciences, Profsoyuznaya st., 84/32, Moscow, 117997, Russia \\ ${ }^{\mathrm{b}}$ Kourovka Astronomical Observatory, Ural Federal University, Lenina st., 51, Ekaterinburg, 620000, Russia
}

\section{A R T I C L E I N F O}

\section{Article history:}

Received 25 July 2012

Received in revised form

6 November 2012

Accepted 13 November 2012

Available online 20 November 2012

\section{Keywords:}

Lunar eclipse

High-resolution spectroscopy

Stratosphere

Water vapor

Ozone

\begin{abstract}
A B S T R A C T
In this paper we present the high-resolution spectral observations of the fragment of lunar surface during the total lunar eclipse of December 10, 2011. The observations were carried out with the fiber-fed echelle spectrograph at the 1.2-m telescope in Kourovka Astronomical observatory (Ural mountains, central Russia). The observed radiation is transmitted by tangent trajectory through the southern polar stratosphere before the reflection from the Moon and the spectra contain a number of absorption bands of atmospheric gases $\left(\mathrm{O}_{2}, \mathrm{O}_{3}, \mathrm{O}_{4}, \mathrm{NO}_{2}, \mathrm{H}_{2} \mathrm{O}\right)$. High-resolution analysis of three $\mathrm{O}_{2}$ bands and $\mathrm{O}_{4}$ absorption effects is used to trace the effective trajectory of solar emission through the stratosphere and to detect the contribution of scattered light. Bands of other gases allow us to measure their abundances along the trajectory.
\end{abstract}

(c) 2012 Elsevier Ltd. All rights reserved.

\section{Introduction}

A lunar eclipse is an astronomical event characterized by a unique geometry of the radiation transfer [1]. Direct solar emission cannot reach the surface of the Moon immersed in the umbra (shadow) of the Earth. But it can be refracted in the Earth's atmosphere and enter the geometrical shadow area. That is why the Moon does not fade in the sky during total eclipses. Along with the refraction, light scattering and absorption take place in the atmosphere. Since these processes can depend on the wavelength, the color of the eclipsed Moon changes and strong atmospheric lines appear in the lunar spectrum. The situation is similar to the spectrum of a distant star of sun-like spectral class during the transit of the planet

\footnotetext{
* Corresponding author. Tel.: +7 91639173 00; fax: + 74953335178 .

E-mail addresses: ougolnikov@gmail.com (O.S. Ugolnikov), punanovaanna@gmail.com (A.F. Punanova), krussh@gmail.com (V.V. Krushinsky).
}

with a dense and optically thick atmosphere [2,3]. In the case of the lunar eclipse, these lines are sensitive to the component concentration near the ray perigee altitude. Normally, tangent transmission spectrum of the Earth's atmosphere can be observed from space, which became the basis of satellite techniques of atmosphere composition measurements [4,5]. Lunar eclipses provide the unique opportunity to carry out such measurements from the ground.

The basis of radiation transfer theory during the lunar eclipses is established in Ref. [1]. Refracted solar emission makes the principal contribution to the brightness of the lunar surface. This simplifies the theory and makes it possible to retrieve the additional aerosol and trace gas extinction in the different layers above the limb [6-8]. However, the emission scattered in the atmosphere can also be noticeable. The theoretical estimation of its contribution is performed in Ref. [9]. It is found to be sufficient for the wavelengths below $450 \mathrm{~nm}$ or in the volcanically perturbed atmosphere, which is not a rare case: the signs of scattered light were found during the 
eclipse of August 16, 2008 [10]. The authors relate these signs to the Kasatochi volcano eruption in Alaska (quite close to the limb) which occurred just before the eclipse.

Photometric analysis of a number of eclipses [6-8] in the red and near-infrared spectral range out of atmospheric gases absorption bands had shown that the brightness of the outer umbra part (less than $0.2^{\circ}$ from the umbra edge) is usually close to the theoretical value for the gaseous atmosphere model without the aerosol extinction, revealing the clear atmosphere conditions above $10 \mathrm{~km}$. The exceptions occur in the equatorial and tropical atmosphere. The most remarkable one is the eastern tropical umbra part illuminated through the South-Asian troposphere during the eclipse of June 15 , 2011, the darkest through the last years [11].

Results of spectral measurements of lunar eclipses are interesting since they are sensitive to the variations of atmospheric components along the tangent path above the limb. This path is long (about several hundreds kilometers), and its sufficient and optically thick fraction lies in the horizontal layer with almost constant altitude. It provides high accuracy and good vertical resolution of limb atmosphere spectroscopy. It is especially true for stratospheric components, first of all, stratospheric ozone. The Chappuis lines of ozone define the general brightness and color characteristics of the eclipsed Moon, although they seem to be quite weak in a transmission spectrum in a vertical atmosphere column.

The spectrum of the lunar surface was obtained with high resolution during the partial eclipse of August 16, 2008 [3], but in the penumbra, where the Moon is partially illuminated by direct solar radiation transmitted above the atmosphere. The umbral area was measured once during the same eclipse in a wide spectral range [2], but with lower spectral resolution (about 1000). In this paper we analyze a number of high-resolution spectra of the lunar surface in the umbra obtained during the total eclipse of December 10, 2011. This time the moonlight did not strongly increase the flux from the surrounding sky background. This eclipse is especially interesting since the solar radiation was transferred through the Antarctic stratosphere not far from the seasonal ozone depression. The long duration and less depth of the eclipse allowed a thorough investigation of the Antarctic stratosphere.

The rest of the paper is organized as follows. Section 2 contains the description of observations. In Section 3 a low-resolution analysis is performed, the atmospheric species with broad absorption bands $\left(\mathrm{O}_{3}, \mathrm{O}_{4}\right.$, and $\left.\mathrm{NO}_{2}\right)$ are studied. High-resolution analysis of $\mathrm{O}_{2}$ lines and trajectory retrieval is presented in Section 4. Investigations of water vapor are discussed in Section 5. Finally, Section 6 contains a conclusion.

\section{Observations}

Spectral observations of the total lunar eclipse of December 10, 2011 were conducted at the Kourovka Astronomical Observatory, Russia $\left(57.0^{\circ} \mathrm{N}, \quad 59.5^{\circ} \mathrm{E}\right)$. The observations were carried out with an optical fiberfed echelle spectrograph at the Nasmyth focus of a $1.2-\mathrm{m}$ telescope. The spectral resolution was about 30,000. The fiber diameter corresponds to the angular size $5^{\prime \prime}$. The instrumental band covers the wavelength range from 410 to $780 \mathrm{~nm}$.

The calibration frames (bias, flat field, ThAr spectrum) were collected once per hour. We used the ThAr lamp spectrum for wavelength scale calibration. The accuracy of this procedure is $0.0003 \mathrm{~nm}$. The narrow lines of the ThAr lamp were used to build the instrument pointspread function (PSF) of the spectrograph. We did not obtain the spectra of the sky because it is not necessary for high-resolution spectra. The sky emission lines are weak during the totality and their flux is negligible (the exposure must be about ten times more than the totality duration to observe the sky lines with a high-resolution spectrograph). All frames were processed with an IRAF/ echelle package [12]. Ambient temperature and humidity were recorded at about five-minute intervals to control the weather condition stability during the observations.

We acquired the spectra of the region of the lunar surface with selenographic coordinates $50^{\circ} \mathrm{S}, 5^{\circ} \mathrm{W}$, southeastwards from bright crater Tycho. This place is characterized by almost uniform albedo. During the eclipse, the place was traversing the southern edge of umbra. The radiation transmitted through the lower Antarctic stratosphere contributed the major portion of brightness of the observed spot. Eight frames (exposure is $300 \mathrm{~s}$ for the each one) were obtained as this surface moved through the umbra. The frames were not combined to improve the signal-to-noise ratio $(\mathrm{S} / \mathrm{N})$. The spectra with eclipsed Moon have the $\mathrm{S} / \mathrm{N}$ ratio up to 65 in different orders, which is sufficient for this study. The $\mathrm{S} / \mathrm{N}$ ratio near the $\mathrm{H} \alpha$ line $(656.3 \mathrm{~nm})$ is about 45 . The observational parameters of the recorded spectra are presented in Table 1 . The positions of the observed spot in the umbra during the exposures are shown in Fig. 1. The limb point was moving eastwards along the Antarctic shore. The midexposure positions of the limb point are depicted in Fig. 2.

To take the lunar albedo spectral dependency into account, we obtained the spectra of the same spot on the lunar surface after the end of the eclipse. The Moon was ascending during the observations. Throughout the eclipse the zenith angle decreased from $70^{\circ}$ to $60^{\circ}$ and after the end of the eclipse it was about $40^{\circ}$. The transparency of the atmosphere over the observatory depends on the wavelength too. To take it into account, the spectra of a standard star were obtained between the

Table 1

Parameters of the observed spectra of the lunar eclipse.

\begin{tabular}{|c|c|c|c|c|}
\hline \multirow[t]{2}{*}{ Spectrum no } & \multicolumn{2}{|c|}{ UT (middle) } & \multirow{2}{*}{$\begin{array}{l}\text { Zenith distance } \\
\text { (deg.) }\end{array}$} & \multirow{2}{*}{$\begin{array}{l}\text { Distance to the } \\
\text { umbra edge } \\
\text { (deg.) }\end{array}$} \\
\hline & $\mathrm{hr}$ & $\min$ & & \\
\hline 1 & 14 & 14.2 & 68.2 & 0.086 \\
\hline 2 & 14 & 20.2 & 67.4 & 0.095 \\
\hline 3 & 14 & 26.3 & 66.7 & 0.101 \\
\hline 4 & 14 & 32.2 & 65.9 & 0.102 \\
\hline 5 & 14 & 38.1 & 65.1 & 0.100 \\
\hline 6 & 14 & 44.4 & 64.3 & 0.093 \\
\hline 7 & 14 & 50.2 & 63.5 & 0.083 \\
\hline 8 & 14 & 56.1 & 62.7 & 0.069 \\
\hline
\end{tabular}




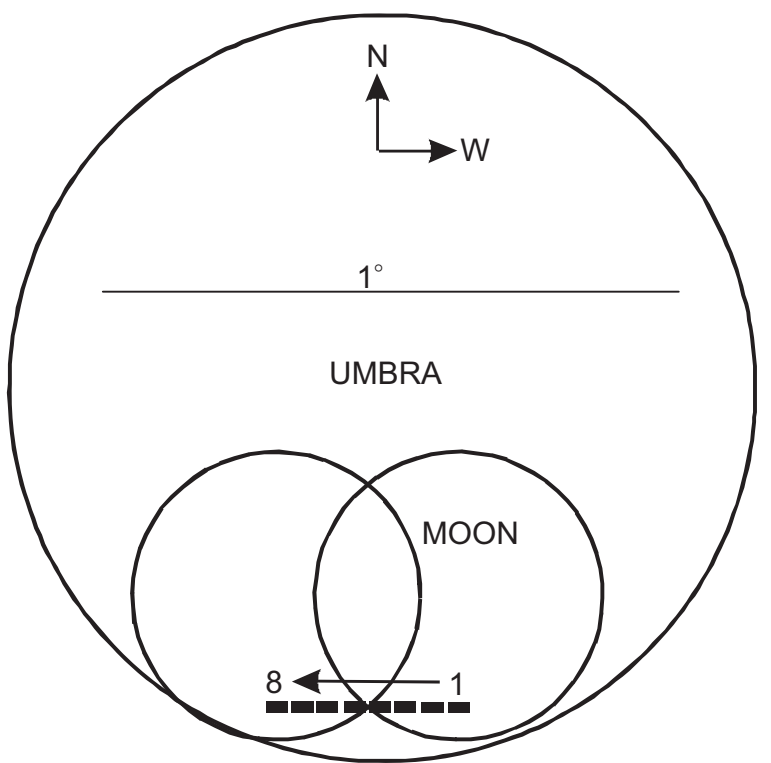

Fig. 1. Positions of the observed point of the lunar surface inside the umbra in the sky (west direction is to the right). The Moon position is shown for the first and the last eclipse frames. The geometrical umbra border (the atmosphere-free case) is not expanded by the factor 1.02 , which is usually done in astronomical ephemeris.

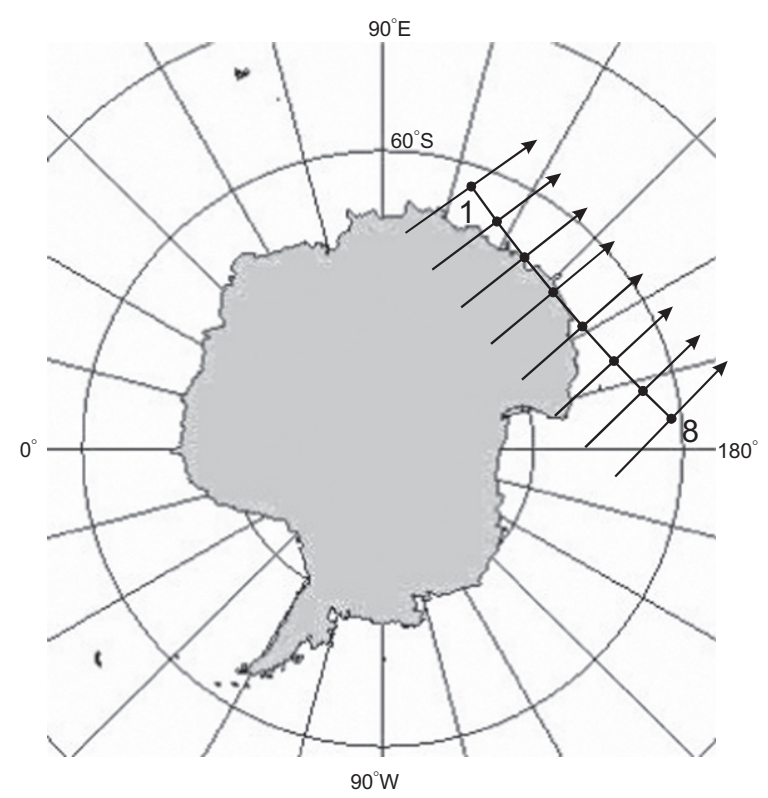

Fig. 2. Motion of the limb point below the light path perigee during the observations. Arrows show the direction of tangent emission propagation.

totality and full Moon observations. The standard star 109 Tauri was close to the Moon during the observational period prior to its lunar occultation. The star has almost the same spectral class as the Sun, but spectral lines of the star are shifted a little due to the star radial velocity (about $+18 \mathrm{~km} / \mathrm{s}$ ).

\section{Spectrum of the tangent limb transmission: $\mathbf{O}_{3}, \mathbf{O}_{4}$, and $\mathrm{NO}_{2}$ absorption}

Far from the range of fine structure atmospheric absorption (first of all, $\mathrm{O}_{2}$ and $\mathrm{H}_{2} \mathrm{O}$ bands) the observed spectrum of the eclipsed Moon can be described by the equation:

$$
F_{\mathrm{E}}(\lambda, z)=S(\lambda) E(\lambda) L(\lambda) A(\lambda)^{M(z)},
$$

where $S$ is the solar spectrum, $E$ is the atmosphere transmission spectrum along the tangent trajectory above the $\operatorname{limb}, L$ is the lunar albedo, $A$ is the local vertical atmosphere transmission spectrum, and $M$ is the atmospheric mass. For zenith angles $z<70^{\circ}$, the last value is equal to $1 / \cos z$. The analogous expression for value $F_{0}(\lambda, z)$ outside the umbra and penumbra has the following form:

$F_{0}(\lambda, z)=S(\lambda) L(\lambda) A(\lambda)^{M(z)}$.

The spectrum of standard star is

$F_{S}(\lambda, z)=C S_{1}(\lambda) A(\lambda)^{M(z)}$

where $S_{1}$ is the natural spectrum of the star and $C$ is the constant defined by the size of star image and its position in the focal plane relative to the fiber. This value varies for different star spectra. If the eclipsed Moon spectrum is obtained at zenith angle $z_{E}$ and the full Moon spectrum is obtained at zenith angle $z_{0}$, then we have:

$E(\lambda)=\frac{F_{E}\left(\lambda, z_{E}\right)}{S(\lambda) L(\lambda) A(\lambda)^{M\left(z_{E}\right)}}=\frac{F_{E}\left(\lambda, z_{E}\right)}{F_{0}\left(\lambda, z_{0}\right)} A(\lambda)^{M\left(z_{0}\right)-M\left(z_{E}\right)}$.

Having two spectra of the standard star at the zenith angles $z_{1}$ and $z_{2}$, we express their ratio:

$\frac{F_{S 1}\left(\lambda, z_{1}\right)}{F_{S 2}\left(\lambda, z_{2}\right)}=\frac{C_{1}}{C_{2}} A(\lambda)^{M\left(z_{1}\right)-M\left(z_{2}\right)}$.

Substituting Eq. (5) into Eq. (4), we have:

$E(\lambda)=C_{12} \frac{F_{E}\left(\lambda, z_{E}\right)}{F_{0}\left(\lambda, z_{0}\right)} \cdot\left(\frac{F_{S 1}\left(\lambda, z_{1}\right)}{F_{S 2}\left(\lambda, z_{2}\right)}\right)^{\frac{M\left(z_{0}\right)-M\left(z_{E}\right)}{M\left(z_{1}\right)-M\left(z_{2}\right)}}$.

here $C_{12}$ is the constant derived from the ratio $\left(C_{1} / C_{2}\right)$. It is also affected by the slant change of lunar albedo due to the difference of phase angles inside and outside the umbra [13]. Due to these factors, there is no absolute radiometric calibration, and the spectrum $E(\lambda)$ can only be found in arbitrary units.

Fig. 3 shows the spectrum $E(\lambda)$ for the middle of totality (position 4 in Fig. 1); all other obtained spectra have the same structure. The general properties are analogous to the spectra of August 2008 eclipse [2,10]. Strong solar spectrum lines (as $\mathrm{H} \alpha$ and $\mathrm{Na}$ doublet) are the same for the eclipse and non-eclipse spectra of the Moon and disappear in the transparency spectrum. The function $E(\lambda)$ increases with the wavelength for $\lambda>600 \mathrm{~nm}$, defining the red color of the eclipsed Moon. The variations, including two minima near $600 \mathrm{~nm}$, are basically related with the spectral profile of ozone Chappuis absorption bands. $\mathrm{NO}_{2}$ bands and effects of $\mathrm{O}_{4}$ absorption around 477 and $577 \mathrm{~nm}$ are also noticeable.

The $\mathrm{O}_{4}$ molecule is the merging product of two $\mathrm{O}_{2}$ molecules and its concentration is known to be proportional to the $\left[\mathrm{O}_{2}\right]$ squared. The absolute values of $\mathrm{O}_{4}$ cross-section 


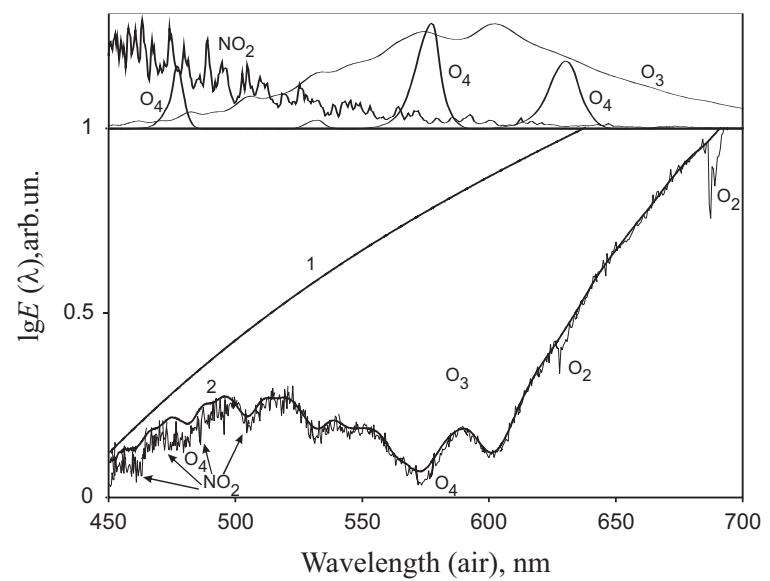

Fig. 3. Transmission spectrum of polar stratosphere in the middle of the eclipse (spectrum 4). The approximation curve 1 corresponds to the non-absorbing gaseous atmosphere, the curve 2 includes the ozone absorption. The upper panel shows the wavelength dependencies of $\mathrm{O}_{3}$ $\mathrm{O}_{4}$, and $\mathrm{NO}_{2}$ cross-sections.

depend on the unknown constants of the merging and dividing reactions, but the absorption spectrum per squared $\mathrm{O}_{2}$ concentration (units of $\mathrm{cm}^{5} /$ molecule $^{2}$ ) is known [14]. Owing to its well-known spatial distribution, $\mathrm{O}_{4}$ is a good tool for the trajectory retrieval and estimation of aerosol and multiple scattering [15]. Since these processes depend on the wavelength, the $\mathrm{O}_{4}$ column densities can vary for different spectral bands. This effect was already noticed in the spectra of the lunar eclipse [10]; however, one of two bands used there $(630 \mathrm{~nm})$ is seen as background behind the $\mathrm{O}_{2}$ lines system and thus hard to study numerically. The $\mathrm{O}_{2}$ bands themselves are better to analyze by highresolution spectroscopy. The results of the analysis are presented below.

To fix the bands of $\mathrm{O}_{3}, \mathrm{O}_{4}$, and $\mathrm{NO}_{2}$, we fit the spectrum by the following model using the least squares method:

$$
\begin{aligned}
\ln E(\lambda)= & \sum_{n=0}^{3} K_{n} \lambda^{n}-Q_{03} S_{O 3}(\lambda)-Q_{N O 2} S_{N O 2}(\lambda)-P_{O 41} n_{O 41}(\lambda) \\
& -P_{042} n_{O 42}(\lambda) .
\end{aligned}
$$

here $s_{\mathrm{O} 3}(\lambda)$ is the cross-section of the ozone molecule and $\mathrm{Q}_{03}$ is the number of $\mathrm{O}_{3}$ molecules per square unit along the emission path. Analogous denotations are made for $\mathrm{NO}_{2}$. The spectrum of the $\mathrm{O}_{4}$ absorption coefficient per squared $\mathrm{O}_{2}$ concentration $n_{\mathrm{O} 4}(\lambda)$ is separated in two parts: the first one is non-zero for shorter wavelengths $\lambda<500 \mathrm{~nm}$, while the second one is for $\lambda>500 \mathrm{~nm}$. The boundary wavelength value $(500 \mathrm{~nm})$ is defined by the total vanishing of $\mathrm{O}_{4}$ absorption. The quantities $P_{\mathrm{O} 41}$ and $P_{\mathrm{O} 42}$ are integrals of $\left[\mathrm{O}_{2}\right]$ squared along the path defined by the 477 and $577 \mathrm{~nm}$ absorption bands of $\mathrm{O}_{4}$, respectively.

The spectral range of $\mathrm{O}_{2}$ and $\mathrm{H}_{2} \mathrm{O}$ absorption bands was not included in this procedure, so the $\mathrm{O}_{4}$ absorption band at $630 \mathrm{~nm}$ (obscured by $\mathrm{O}_{2}$ lines) was not actually taken into account. The values of cross-sections of $\mathrm{O}_{3}$ and $\mathrm{NO}_{2}$ for polar stratosphere conditions are taken from Refs, [16] and [17], respectively. The result of the procedure is also shown in Fig. 3. The ozone absorption profile fits the

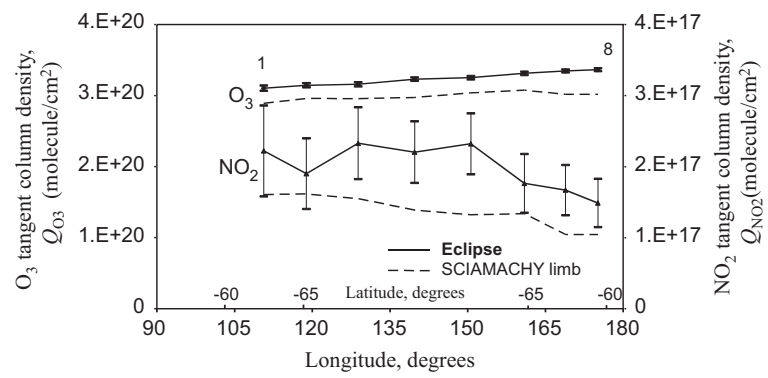

Fig. 4. Tangent column densities of $\mathrm{O}_{3}$ and $\mathrm{NO}_{2}$ along the effective path for different point positions inside the umbra compared with SCIAMACHY limb data.

observed spectral features of $E(\lambda)$ except the $\mathrm{O}_{2}$ bands in the right, $\mathrm{O}_{4}$ and $\mathrm{NO}_{2}$ bands add just a little correction visible in the figure. The resulting value of $P_{\mathrm{O} 41}$ (about $10^{44}$ molecule ${ }^{2} / \mathrm{cm}^{5}$ in the middle of totality) is surely about 2 times higher than $P_{\mathrm{O} 42}\left(5 \cdot 10^{43}\right.$ molecule $\left.{ }^{2} / \mathrm{cm}^{5}\right)$, showing the different radiation transfer geometry at 477 and $577 \mathrm{~nm}$ and the scattered light contribution in the first of these bands. This question will be addressed below (Section 4).

The ozone Chappuis absorption is found to be strong. Comparing the curves with and without ozone (the same figure), we see that the lunar surface brightness at $600 \mathrm{~nm}$ is reduced by a factor of 6 . The dependences of $\mathrm{O}_{3}$ and $\mathrm{NO}_{2}$ tangent column densities on the coordinates of the Antarctic limb points are shown in the Fig. 4. The changes of $\mathrm{NO}_{2}$ abundance seem to be related to its diurnal variations. The first spectra correspond to the evening part of the limb (Sun is setting), the last spectra-to the morning one (Sun is rising). The number of $\mathrm{NO}_{2}$ molecules naturally anti-correlates with the number of $\mathrm{O}_{3}$ molecules. The accuracy of the estimation of $\mathrm{NO}_{2}$ abundance is not so good. The reasons for that are the low intensity of eclipsed Moon in the blue part of the spectrum where $\mathrm{NO}_{2}$ absorption takes place, and the influence of scattered light for $\lambda<500 \mathrm{~nm}$.

The relative accuracy of the ozone abundance determination is much better. We see that ozone concentration in the lower stratosphere is slowly increasing eastwards. It partially agrees with the behavior of total ozone column density by SCIAMACHY TOSOMI data [18]. To compare the trajectory results with available satellite data on the same day, the retrieval of trajectory parameters is needed, which will be done below.

\section{Effective trajectory retrieval}

Fig. 5 shows the principal scheme of radiation transfer during the lunar eclipse. For each moment, the position of the Earth's limb can be calculated. Given the position angle of the lunar surface element in the umbra, we find the coordinates of the limb point lying in the plane including the lunar surface point being measured and the centers of the Earth and the Sun. It is the perigee projection on the surface of the Earth.

Solar emission crosses the atmosphere twice (paths 1 and 2 in the figure). The object of interest is the fraction of 


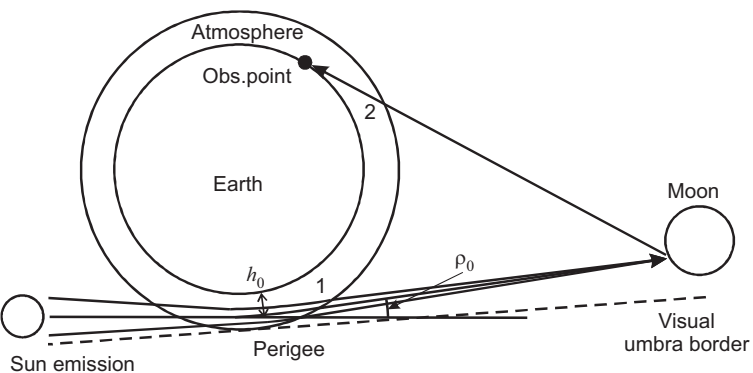

Fig. 5. Scheme of radiation transfer during the lunar eclipse.

tangent trajectory path through the southern polar atmosphere (path 1 ). It is characterized by the perigee altitude $h_{0}$ or the corresponding pressure $p_{0}$. Given the model of vertical temperature distribution, we can calculate the refraction angle $\rho_{0}$ for this path.

The lunar surface observation point was about $0.1^{\circ}$ inside the umbra edge. Adding the solar angular radius, we can expect that the effective refraction angle is not more than $0.3-0.4^{\circ}$ (the value can be also decreased by the aerosol extinction in the lower atmosphere), showing that the effective trajectory perigee lies in the lower stratosphere. The temperature does not sufficiently vary with altitude there. The characteristic temperature for this season and location $(220 \mathrm{~K})$ is based on the MIMOSA data [19]. The atmosphere absorption takes place mostly along the lowest trajectory fraction near the perigee with the altitudes just a little bit more than the perigee altitude $h_{0}$. Characterizing the path by the perigee pressure $p_{0}$, we can use the isothermal model of the lower stratosphere. To calculate the transmission spectrum, we assume the path to have the constant pressure $p_{0}$ and the length giving the same number of molecules along the path. This length can be calculated by the formula

$D_{0}=B \sqrt{2 \pi R H}$.

here $R$ is the Earth's radius, and $H$ is the atmospheric scale height for the temperature $220 \mathrm{~K}$. The factor $B$ slightly exceeds unity and is characterized by the curvature of the light trajectory. It depends on the perigee pressure, being close to 1.1 for the lower stratosphere.

The effective trajectory of radiation transfer can be retrieved by the spectral lines of the atmospheric component with well-known spatial distribution, such as molecular oxygen $\mathrm{O}_{2}$. The instrumental range includes three $\mathrm{O}_{2}$ absorption bands with the wavelengths 630,690 , and $765 \mathrm{~nm}$. Taking the parameters of each line from the HITRAN database [20] and building the line profiles using the SPECTRA system [21] with account of PSF of our detector $Q\left(\lambda, \lambda_{i}\right)$, we can calculate the spectrum of the eclipsed Moon in each of three bands:

$$
\begin{aligned}
F_{E L}(\lambda)= & \int_{-\lambda_{P S F}}^{\lambda_{P S F}} S\left(\lambda+\lambda_{i}\right) E_{L}\left(p_{0}, D_{0}, \lambda+\lambda_{D}+\lambda_{i}\right) \\
& \times L\left(\lambda+\lambda_{i}\right) A_{L}\left(z, \lambda+\lambda_{i}\right) Q\left(\lambda, \lambda_{i}\right) d \lambda_{i} .
\end{aligned}
$$

The definitions are analogous to Eq. (1), $E_{L}$ and $A_{L}$ are the atmospheric transmission spectra in $\mathrm{O}_{2}$ bands by trajectories 1 and 2, respectively. Due to the fine structure of the lines at the scale comparable or narrower than the instrumental profile, the eclipsed Moon spectrum in the $\mathrm{O}_{2}$ bands cannot be considered as the simple multiplication of transmission spectra by the paths 1 and 2 (as was done above for $\mathrm{O}_{3}, \mathrm{O}_{4}$, and $\mathrm{NO}_{2}$ ) and must be built by convolution of both transmission spectra and instrumental band $Q\left(\lambda, \lambda_{i}\right)$. We also take into account the tiny Doppler shift of transmission spectra $\left(\lambda_{D}\right)$. This shift is due to the double radial velocity of the Moon and motion of the observer towards the Moon together with the rotating surface of the Earth. The combined effect has the value about $0.4 \mathrm{~km} / \mathrm{s}$. It is less than instrumental resolution but comparable with fine structure scale of $\mathrm{O}_{2}$ spectrum and, thus, slightly changes the final result.

The $\mathrm{O}_{2}$ transmission spectrum of atmospheric layer above the observer, $A_{L}$, is defined by the zenith angle of the Moon $z$ and total amount of $\mathrm{O}_{2}$ in the vertical atmospheric column, and slightly depends on the distribution of the troposphere temperature. Since the relative abundance of $\mathrm{O}_{2}$ does not vary with altitude in the troposphere, the spectrum can be easily integrated by the atmosphere layers with equal step in pressure value.

Formula (9) is used to run the best-fit procedure to derive the effective perigee pressure $p_{0}$ of the path through the polar stratosphere. To do this, we take the solar spectrum $S(\lambda)$ from Ref. [22] and consider the spectral dependencies of lunar albedo $L(\lambda)$, atmosphere absorption (including $\mathrm{O}_{3}$ and $\mathrm{O}_{4}$ bands) and scattering to be polynomial (second degree) within each of three narrow ranges of $\mathrm{O}_{2}$ absorption.

Another way to retrieve the effective radiation path through the polar atmosphere is $\mathrm{O}_{4}$ absorption discussed in the previous chapter which gives the integral values of $\left[\mathrm{O}_{2}\right]$ squared along the path. This procedure adds two more spectral intervals (477 and $577 \mathrm{~nm}$ ) expanding the analysis to the greater part of the observed spectral range. The dependence of the pressure $p_{0}$ on $\mathrm{O}_{4}$ abundance is quite weak due to the rapid decrease of the latter value with the altitude, so we can estimate $p_{0}$ based even on a poorly-defined $\mathrm{O}_{4}$ absorption.

Fig. 6 displays the eclipsed Moon spectrum $\mathrm{O}_{2}$ bands in the range from 759 to $766 \mathrm{~nm}$ compared with the best-fit curve and the one for the uneclipsed case at the same zenith distance. The results of the best-fit procedure for

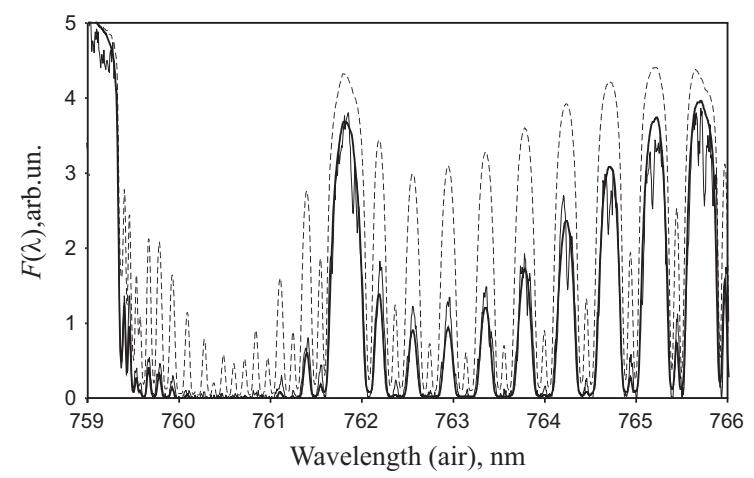

Fig. 6. Fragment of spectrum of the lunar surface 4 with $\mathrm{O}_{2}$ bands near $765 \mathrm{~nm}$. The solid line is the best-fit model, the dashed one corresponds to the local atmosphere absorption only. 


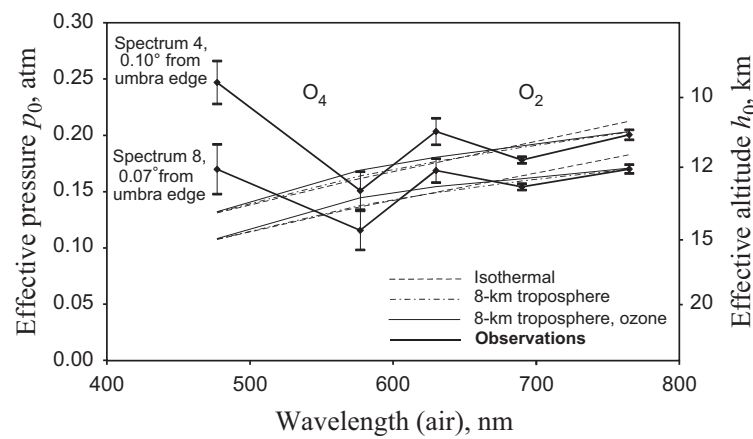

Fig. 7. Wavelength dependency of effective ray perigee pressure and altitude (scale for 8 -km troposphere model) by $\mathrm{O}_{2}$ and $\mathrm{O}_{4}$ bands observations (spectra 4 and 8 ) compared with different atmosphere aerosol-free models.

three systems of $\mathrm{O}_{2}$ lines are shown in the Fig. 7. The typical values of the effective pressure for these bands are about 0.16-0.20 atm. The corresponding altitude $(11-12 \mathrm{~km})$ is above the Antarctic tropopause level. The results of $\mathrm{O}_{4}$ analysis are shown in the same figure.

Fig. 7 presents the results of spectra 4 and 8, obtained at maximal and minimal angular distances from the umbra border, respectively. The behavior of $p_{0}$ values for other spectra is quite regular: the value increases as the point immerses into umbra and decreases after the middle of totality. It was expected since the refraction angle increases with the perigee pressure.

The figure also contains theoretical dependencies of the same value. The first of these dependencies is calculated for the isothermal ozone-free atmosphere with temperature $220 \mathrm{~K}$. The second model also contains $8-\mathrm{km}$ troposphere with linear temperature gradient $-5 \% \mathrm{~km}$ and ground temperature $260 \mathrm{~K}$ (the shift of the stratosphere altitude-pressure dependencies between these models is about $0.7 \mathrm{~km}$ ). The third theoretical curve corresponds to the model with ozone Chappuis absorption for the typical ozone vertical distribution for this date and location based on SCIAMACHY limb measurements $[5,23]$.

One can see that all model results are quite close to each other, showing a small influence of troposphere temperature variations and even ozone absorption on the $p_{0}$ value. The theoretical curves are also close to the observational ones for all wavelengths except $477 \mathrm{~nm}$. This suggests the lack of aerosol extinction in the whole range of altitudes, where the radiation of different Sun points is refracted ( $8 \mathrm{~km}$ and above). This extinction would decrease the contribution of deeply immersed parts of the Sun with strong absorption lines and, thus, weaken these lines in the observed spectrum and decrease the $p_{0}$ value. However, this effect was not observed.

In the $477 \mathrm{~nm} \mathrm{O}_{4}$ band the reverse effect takes place-the effective pressure $p_{0}$ exceeds the gaseous value, especially deep inside the umbra (upper curve in Fig. 7). The effect was found above as the increased $P_{\mathrm{O} 41}$ value. As it was shown theoretically [9], the scattered (Rayleigh and aerosol) light contribution becomes noticeable at such wavelengths (or even at longer ones if the atmosphere is volcanically perturbed, but it is not the case of December 2011 eclipse). The trajectory of scattered light varies depending on the location of scattering point. $\mathrm{O}_{4}$ analysis shows that the optical path of scattered light is longer than the one for refracted light, and the scattering trajectory lies below or (most probably) aside the refracted trajectory (however, theoretical analysis [9] had shown that the effective scattering takes place higher). The path can be longer or reach lower atmospheric layers with increased concentration of $\mathrm{O}_{2}$ and, especially, $\mathrm{O}_{4}$. Composition of both effects leads to the sufficient shift of $p_{0}$ in Fig. 7.

The typical value of $p_{0}$ for the range of Chappuis ozone bands is about $0.17 \mathrm{~atm}$, and the corresponding altitude $h_{0}$ is $12 \mathrm{~km}$. It allows us to compare the obtained $\mathrm{O}_{3}$ and $\mathrm{NO}_{2}$ molecule numbers with SCIAMACHY limb measurements $[5,23,24]$ for the same date, integrating the data along the trajectory. The results are shown by the dashed lines in Fig. 4. According to our observations during the eclipse, the abundance of ozone is higher than the same abundance from SCIAMACHY data. It can be explained by the accuracy of estimation of both our measurements (especially for $\mathrm{NO}_{2}$ ) and SCIAMACHY data, where the relative error of $\left[\mathrm{O}_{3}\right]$ and $\left[\mathrm{NO}_{2}\right]$ near $10 \mathrm{~km}$ is about several dozens of percents.

\section{Water vapor measurements}

The observed spectral range covers the $\mathrm{H}_{2} \mathrm{O}$ absorption bands around $730 \mathrm{~nm}$. This band consists of a number of strong separated lines and can be used to measure the tangent column density of $\mathrm{H}_{2} \mathrm{O}$ along the emission path. The absorption by water vapor takes place at both atmospheric fractions of the path-southern polar stratosphere and troposphere above the observatory.

Fig. 8 shows the fragment of the lunar surface spectra near $730 \mathrm{~nm}$ inside and outside the umbra. In the eclipsefree case, just the local troposphere contributes to the absorption. The $\mathrm{H}_{2} \mathrm{O}$ lines are naturally weaker than during the eclipse, but the analysis shows the similar line profile characteristics, typical for higher atmosphere levels at lower pressure. The possible reason for that is current special properties of water vapor distribution above the observatory during the observations.

This circumstance allows us to simplify the numerical analysis. We consider the line profile to be the same along

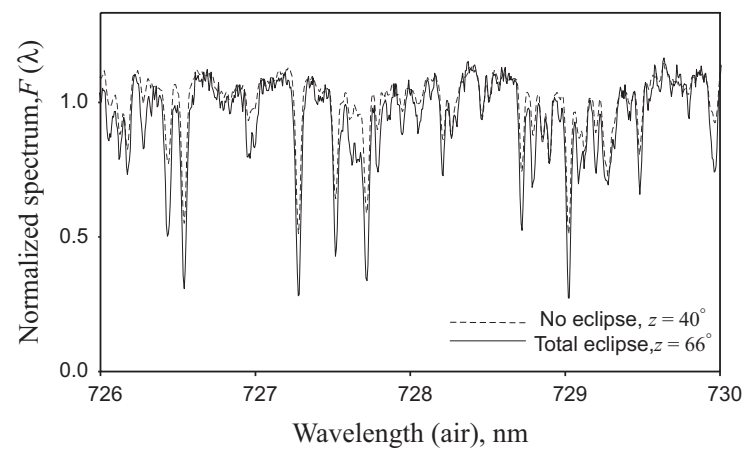

Fig. 8. Fragment of lunar surface spectra inside (spectrum 4) and outside the umbra with $\mathrm{H}_{2} \mathrm{O}$ bands near $730 \mathrm{~nm}$. 
the whole path. Since we are interested mainly in the polar stratosphere and have to separate corresponding $\mathrm{H}_{2} \mathrm{O}$ absorption, we take the HITRAN profile [20,21] for the temperature $T_{0}(220 \mathrm{~K})$ and the effective pressure $p_{0}$ obtained in the previous section for this spectral range, which is $0.18 \mathrm{~atm}$. The spectrum corresponding to the $\mathrm{H}_{2} \mathrm{O}$ effective path length $L_{P}(z)$ is convolved with the instrumental profile

$$
\begin{aligned}
F_{E L}(\lambda)= & \int_{-\lambda_{P S F}}^{\lambda_{P S F}} S\left(\lambda+\lambda_{i}\right) L\left(\lambda+\lambda_{i}\right) E(\lambda) A(\lambda, z) \\
& \times \exp \left(-X_{H 2 O}\left(p_{0}, T_{0}, \lambda+\lambda_{i}\right) \cdot L_{P}(z)\right) Q\left(\lambda, \lambda_{i}\right) d \lambda_{i} .
\end{aligned}
$$

here $E(\lambda)$ and $A(\lambda, z)$ are the continuum light extinction by the paths 1 and 2 (that may be considered as polynomial), $X_{\mathrm{H} 2 \mathrm{O}}$ is the water cross-section integrated using the HITRAN database. $L_{P}(z)$ is to be found by the least squares method by comparison with observational data. To exclude the local troposphere contribution, we have to run this procedure not only for the umbral spectra, but also for full Moon and standard star ones, covering the wide range of zenith angles of the source.

The results can be seen in the bottom of Fig. 9. Observations of the uneclipsed Moon and standard star cover the range of atmospheric masses from 1.3 to 2.0. For all these spectra the effective $\mathrm{H}_{2} \mathrm{O}$ path length (or corresponding slant column density) is proportional to the atmospheric mass (or $1 / \cos z$ ). It shows the stable local condition of water vapor during the observations. The value of vertical $\mathrm{H}_{2} \mathrm{O}$ column density above the observatory is found to be $0.47 \mathrm{~g} / \mathrm{cm}^{2}$, corresponding to the length $L_{0} 26.1 \mathrm{~m}$ for the pressure and temperature noted above. The local $\mathrm{H}_{2} \mathrm{O}$ vertical column density slightly exceeds the SCIAMACHY AMC-DOAS data value for this region ([25], $0.34 \mathrm{~g} / \mathrm{cm}^{2}$ ). The effective length of the stratosphere water path can be found using the Bouger's law:

$L_{s}=L_{P}(z)-\frac{L_{0}}{\cos z}$

Dependency of this value is also shown in Fig. 9. Typical $L_{S}$ amount for most part of the recorded spectra is $15 \mathrm{~m}$ that corresponds to the tangent column density $0.27 \mathrm{~g} / \mathrm{cm}^{2}$ for the pressure $0.18 \mathrm{~atm}$ and temperature $220 \mathrm{~K}$. The $\mathrm{H}_{2} \mathrm{O}$ molecule number slightly increases in the western part of the limb, where the ozone abundance along the same path decreases.

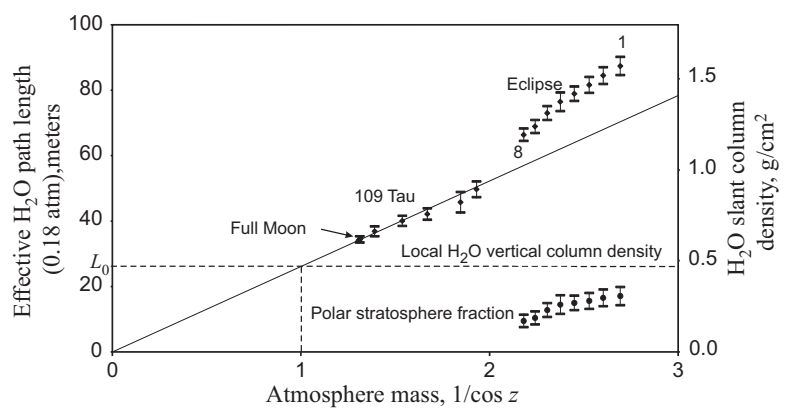

Fig. 9. Abundance of $\mathrm{H}_{2} \mathrm{O}$ in the polar stratosphere and local atmosphere based on the observations.
Given the $\mathrm{H}_{2} \mathrm{O}$ vertical column density by SCIAMACHY AMC-DOAS data [25] for the most part of the limb locations (about $0.6 \mathrm{~g} / \mathrm{cm}^{2}$ ), we estimated the characteristic scale height of $\mathrm{H}_{2} \mathrm{O}$ distribution by the method described in Ref. [7]. The obtained amount is about $2.0 \mathrm{~km}$. It is higher than the tropical latitude value by the eclipse of March 4, 2007, but the stratosphere $\mathrm{H}_{2} \mathrm{O}$ abundance and the scale value obtained in that photometric paper (1.3 km, [7]) seem to be underestimated due to the Forbes effect (underestimation of fine structure absorption by wide spectral band photometry) caused by the structure of $\mathrm{H}_{2} \mathrm{O}$ bands. High-resolution spectroscopy allows us to improve the accuracy of the scale value. If the $\mathrm{H}_{2} \mathrm{O}$ distribution followed the exponential law with the same scale both in the troposphere and stratosphere, then relative $\mathrm{H}_{2} \mathrm{O}$ abundance in the southern lower stratosphere would be found to be about $50 \mathrm{ppm}$. However, as we saw for the observatory location, the $\mathrm{H}_{2} \mathrm{O}$ distribution with altitude does not follow this exponential law, and this amount is just the rough estimation that overestimates the value above the tropopause.

\section{Conclusions and perspectives}

In this paper the analysis of high-resolution spectra of the Moon during the lunar eclipse was performed. The scheme of radiation transfer provides the opportunity to study the absorption of the trace gases along the tangent path numerically far from the place where observations were carried out. Usually, this can be done only by space observations. The position of effective path versus the wavelength allows us to estimate the contribution of scattering emission. It is found to be small at the wavelengths above $500 \mathrm{~nm}$. At this spectral range the eclipsed Moon is quite bright and a large number of atmospheric absorption bands appears.

The lunar eclipse spectra were obtained for the wavelengths up to $780 \mathrm{~nm}$, including $\mathrm{O}_{2}$ and $\mathrm{H}_{2} \mathrm{O}$ bands. However, if it is expanded to the IR-range, the lines of greenhouse gases $\mathrm{CO}_{2}$ and $\mathrm{CH}_{4}$ will be included in the analysis [2]. By increasing the number of points of lunar surface or by using the spectrograph with the slit oriented along the umbra radius, we can analyze different paths simultaneously and study the vertical distribution of optically active gases.

\section{Acknowledgments}

The authors would like to thank Andrey Sobolev, Artem Burdanov, Alexander Popov and Konstantin Mironov (Kourovka Astronomical Observatory) for their help in preparation and conducting of observations. We also thank Christian von Savigny and Stefan Noël (Institute of Environmental Physics/Remote Sensing, University of Bremen, Germany) for providing the $\mathrm{O}_{3}, \mathrm{NO}_{2}$ and $\mathrm{H}_{2} \mathrm{O}$ SCIAMACHY data, Boris Voronin, Semen Mikhailenko (Institute of Atmosphere Optics, Tomsk, Russia) and Oleg Postylyakov (Institute of Atmospheric Physics, Moscow, Russia) for the help on working with SPECTRA system and the HITRAN database. 
The work was performed with partial support by Russian Federal program "Investigations and Elaborations on Priority courses of Russian Scientific and Technological Complex Development 2007-2012" (State contract no 16.518.11.7074) and Russian Foundation for Basic Research (grant no 12-05-00501).

\section{References}

[1] Link F. Die mondfinsternisse (lunar eclipses). Leipzig: Akademische Verlagsgesellschaft; 1956.

[2] Palle E, Zapatero Osorio MR, Barrena R, Montanes-Rodriguez P, Martin EL. Earth's transmission spectrum from lunar eclipse observations. Nature 2009;459:814-6.

[3] Vidal Madjar A, et al. The Earth as an extrasolar transiting planet. Earth's atmospheric composition and thickness revealed by lunar eclipse observations. Astron Astrophys 2010;523:A57-71.

[4] MacCormick MP, Chu WP, Grams GW. High-latitude stratospheric aerosols measured by SAM-II satellite system in 1978 and 1979 Science 1981;214:328-31.

[5] Noël S, Bovensmann H, Burrows JP, Frerick J, Chance KV, Goede AHP. Global atmospheric monitoring with SCIAMACHY. Phys Chem Earth 1999;24:427-34.

[6] Ugolnikov OS, Maslov IA. Atmospheric aerosol limb scanning based on the lunar eclipses photometry. J Quant Spectrosc Radiat Transfer 2006;102:499-512.

[7] Ugolnikov OS, Maslov IA. Altitude and latitude distribution of atmospheric aerosol and water vapor from the narrow-band lunar eclipse photometry. J Quant Spectrosc Radiat Transfer 2008;109: 378-88.

[8] Ugolnikov OS, Maslov IA. Remote sensing of the earth atmosphere based on the lunar eclipses observations. Atmos Oceanic Opt 2009;22:365-9.

[9] Garcia Munoz A, Palle E. Lunar eclipse theory revisited: Scattered sunlight in both the quiescent and the volcanically perturbed atmosphere. J Quant Spectrosc Radiat Transfer 2011;112:1609-21.

[10] Garcia Munoz A, Palle E, Zapatero Osorio MR, Martin EL. The impact of the Kasatochi eruption on the Moon's illumination during the August 2008 lunar eclipse. Geophys Res Lett 2011;38:L14805-9.

[11] Ugolnikov OS, Maslov IA, Korotkiy SA. Spectral and spatial dependency of aerosol extinction in the upper troposphere based on the moon surface photometry in the earth's umbra. Cosm Res 2012;50: $340-5$.

[12] Tody D, IRAF in the Nineties. astronomical data analysis software and systems II, A.S.P. Conference Series. Hanisch RJ, Brissenden RJV, Jeannette Barnes, eds. 1993; 52:173.
[13] Lane AP, Irwine WM. Monochromatic phase curves and albedos for the lunar disk. Astron J 1973;78:267-77.

[14] Greenblatt GD, Orlando JJ, Burkholder JB, Ravishankara AR. Absorption measurements of oxygen between 330 and $1140 \mathrm{~nm}$. J Geophys Res 1990;95:18577-82.

[15] Wagner T, Dix B, von Friedeburg C, Frieß U, Sanghavi S, Sinreich R, et al. MAX-DOAS $\mathrm{O}_{4}$ measurements: A new technique to derive information on atmospheric aerosols - Principles and information content. J Geophys Res 2004;109:D22205-25.

[16] Voigt S, Orphal J, Bogumil K, Burrows JP. The temperature dependence $(203-293 \mathrm{~K})$ of the absorption cross-sections of $\mathrm{O}_{3}$ in the 230-850 nm region Measured by Fourier-transform spectroscopy. J Photochem Photobiol, A: Chem 2001;143:1-9.

[17] Voigt S, Orphal J, Burrows JP. High-resolution reference data by UV-visible Fourier-transform spectroscopy: 1. Absorption crosssections of $\mathrm{NO}_{2}$ in the $250-800 \mathrm{~nm}$ range at atmospheric temperatures (223-293 K) and pressures (100-1000 mbar). Chem Phys Lett 2012 in press.

[18] Eskes HJ, van der A RJ, Brinksma EJ, Veefkind JP, de Haan JF, Valks PJM. Retrieval and validation of ozone columns derived from measurements of SCIAMACHY on Envisat. Atmos Chem Phys Discuss 2005;5:4429-75.

[19] Hauchecorne A, Godin S, Marchand M, Heese B, Souprayen C. Quantification of the transport of chemical constituents from the polar vortex to middle latitudes in the lower stratosphere using the high-resolution advection model MIMOSA and effective diffusivity. J Geophys Res 2002;107:D20-32.

[20] Rothman LS, Gordon IE, et al. The HITRAN 2008 molecular spectroscopic database. J Quant Spectrosc Radiat Transfer 2009; 110:533-72.

[21] Mikhailenko SN, Babikov YuL, Golovko VF. Information-calculating system Spectroscopy of Atmospheric Gases. The structure and main functions. Atmos Oceanic Opt 2005;18:685-95.

[22] Wallace L, Hinkle K, Livingston W. An atlas of the spectrum of the solar photosphere from 13,500 to $33,980 \mathrm{~cm}^{-1}$ (2942 to 7405 Angstroms). N.S.O. Tech. Rept 2007 \#07-001.

[23] Sonkaew T, Rozanov VV, von Savigny C, Rozanov A, Bovensmann H, Burrows JP. Cloud sensitivity studies for stratospheric and lower mesospheric ozone profile retrievals from measurements of limbscattered solar radiation. Atmos Meas Tech 2009;2:653-78.

[24] Rozanov A, Bovensmann H, Bracher A, Hrechanyy S, Rozanov V, Sinnhuber $\mathrm{M}$, et al. $\mathrm{NO}_{2}$ and $\mathrm{BrO}$ vertical profile retrieval from SCIAMACHY limb measurements: Sensitivity studies. Adv Space Res 2005;36:846-54.

[25] Noël S, Buchwitz M, Bovensmann H, Burrows JP. Validation of SCIAMACHY AMC-DOAS water vapour columns. Atmos Chem Phys 2005;5:1835-41. 